\title{
Charmonium production in heavy ion collisions
}

\author{
N.S. Topilskaya* and A.B. Kurepin \\ Institute for Nuclear Research, Moscow \\ E-mail: topilska@inr.ru, kurepin@inr.ru
}

Measurment of lepton pairs is a useful tool for investigation the properties of hot and dense matter created in heavy-ion collisions. The review of the experimental data on charmonium states production via their decay on lepton pairs measured at CERN SPS and at LHC and comparison with the data obtained at RHIC is presented. The suppression of $\mathrm{J} / \psi$ production may be sensitive to a possible quark-gluon plasma (QGP) formation. The anomalous suppression of $\mathrm{J} / \psi$ production at the CERN SPS was discovered in central Pb-Pb collisions by NA50 collaboration at $158 \mathrm{GeV}$. However, the effects of $\mathrm{J} / \psi$ suppression in cold nuclear matter and feed-down production from higher charmonium states are important for $\mathrm{J} / \psi$ production at SPS energies. The PHENIX experiment at RHIC at $\sqrt{s}=200 \mathrm{GeV}$ shows that the $\mathrm{J} / \psi$ suppression for $\mathrm{Au}-\mathrm{Au}$ collisions as a function of multiplicity is of the same order as the suppression at SPS energies for $\mathrm{Pb}-\mathrm{Pb}$. The study of charmonium production in $\mathrm{Pb}-\mathrm{Pb}$ collisions by ALICE experiment at LHC gives less suppression than at RHIC and indicates on the possibility of the $\mathrm{J} / \psi$ regeneration via $c \bar{c}$ recombination process. The contribution of B-decay should be taken into account at LHC energy. The high statistic measurements at LHC could investigate the properties of matter at high energy density and temperature. Also the energy interval between SPS and RHIC is very important for study of the mechanism of quarkonium production and suppression, in order to investigate medium effects and conditions for quark-gluon plasma formation. However, the luminosity in collider experiments at RHIC is decreased with energy reduction. If the proton and ion beams will be used at LHC with fixed targets, the energy interval between SPS and RHIC in p-A and A-A collisions could be investigated. For $7 \mathrm{TeV}$ proton beam, the energy in N-N c.m. $\sqrt{s}=114.6 \mathrm{GeV}$, for 2.75 $\mathrm{TeV} \mathrm{Pb}$ beam $\sqrt{s}=71.8 \mathrm{GeV}$. This is unique possibility to clarify the mechanism of charmonium production since the probability of $c \bar{c}$ recombination is decreased by diminution of the collision energy.

XXI International Baldin Seminar on High Energy Physics Problems September 10-15, 2012

JINR, Dubna, Russia

\footnotetext{
*Speaker.
} 


\section{Introduction}

For investigation the properties of hot and dense matter created in heavy-ion collisions the measurment of lepton pairs is a usefull tool. For different beam energies in heavy?ion collisions the temperature and baryonic chemical potential are changed. The review of the experimental data on charmonium states production via their decay on lepton pairs measured at the CERN SPS and in p-p and $\mathrm{Pb}-\mathrm{Pb}$ collisions at $\mathrm{LHC}$ and comparison with the data obtained at the Brookhaven National Laboratory Relativistic Heavy Ion Collider RHIC is presented. Heavy quarkonia are sensitive to the collision dynamics and the suppression of charmonium $J / \psi$ production was suggested as a possible signal of quark-gluon plasma (QGP) formation. But the experimental and theoretical situation is more complicated. At the CERN SPS the "anomalous" suppression of $\mathrm{J} / \psi$ production was discovered in central $\mathrm{Pb}-\mathrm{Pb}$ collisions at $158 \mathrm{GeV}$ by NA50 collaboration. But the effects of $\mathrm{J} / \psi$ suppression in cold nuclear matter and feed-down production from higher charmonium states are important in production of $\mathrm{J} / \psi$ at SPS energies.

At RHIC in the PHENIX experiment at $\sqrt{s}=200 \mathrm{GeV}$ for Au-Au collisions it was found that the $\mathrm{J} / \psi$ suppression as a function of multiplicity is of the same order as the suppression at SPS for $\mathrm{Pb}-\mathrm{Pb}$. However, the suppression measured by PHENIX is stronger than suppression at SPS if the dependence of "anomalous" J/ $\psi$ suppression on number of participants, $N_{\text {part }}$, is considered.

The study of charmonium production at LHC shows less suppression of $\mathrm{J} / \psi$ than at RHIC and points out on the importance of regeneration process. At LHC energy the contribution of B-decay to $\mathrm{J} / \psi$ should be taken into account.

To understand the properties of nuclear matter it is important to have experimental data also at low energy. Unfortunately, beam energy scan at RHIC produced data with low statistic and large systematic errors. It is planned to study the mechanism of quarkonium production and suppression up to $35 \mathrm{GeV} /$ per nucleon (SIS300) at the FAIR in CBM and at NICA collider in Dubna with high statistic. The energy interval between SPS and RHIC could be investigated using LHC proton and ion beams with fixed targets. The data could be used for the study of the mechanism of quarkonium production and suppression, in order to investigate medium effects and conditions for quark-gluon plasma formation, since the probability of $c \bar{c}$ recombination decreases with reduction of the energy of collision. In order to understand the problem of quarkonium suppression it is necessary to measure p-p, p-A and A-A collisions in the same kinematical domain.

\section{Charmonium production at the CERN SPS}

The suppression of $\mathrm{J} / \psi$ production by colour screening in a dense nuclear matter was suggested as one of the possible signature of quark-gluon plasma formation in relativistic heavy ions collision [1]. The charmonium production was measured by NA50 experiment in $\mathrm{Pb}-\mathrm{Pb}$ collisions at 158 $\mathrm{GeV}$ per nucleon [2] and in p-A collisions at 400 and $450 \mathrm{GeV}$ [3]. It was observed the normal nuclear suppression of $\mathrm{J} / \psi$ in proton-nucleus reactions and "anomalous" suppression near $40 \%$ in central lead-lead collisions. The cross section for normal nuclear absorption in $\mathrm{Pb}-\mathrm{Pb}$ collisions was obtained from p-A data by assumption of a weak energy dependence of the cross section. Then $\mathrm{J} / \psi$ production in p-A collisions at 400 and $158 \mathrm{GeV}$ and in In-In collisions at $158 \mathrm{GeV}$ per nucleon was measured by NA60 experiment at SPS. It was found rather strong energy dependence of the absorption cross section. Therefore it is very important to measure charmonium production cross 
section in p-p, p-A and A-A collisions in the same kinematical domain [4]. The suppression of $\mathrm{J} / \psi$ production in cold nuclear matter (CNM) include not only charmonium absorption in the final state, but also initial state effects. First of all it is the shadowing effect, i.e. the modification of the parton distribution function in nucleus in comparison to the nucleon. Also, it is necessary to take into account the energy loss of the parton traversing the nucleus before the hard scattering. The existing shadowing parameterizations depend on the model assumptions for nuclear parton distribution. Using EKS98 shadowing parameterization [5] the extracted absorption cross section is increased. For In-In collisions at $158 \mathrm{GeV}$ per nucleon the "anomalous" suppression in central collisions is rather small, while for central lead-lead collisions the value of "anomalous" suppression remains near $20-30 \%$.

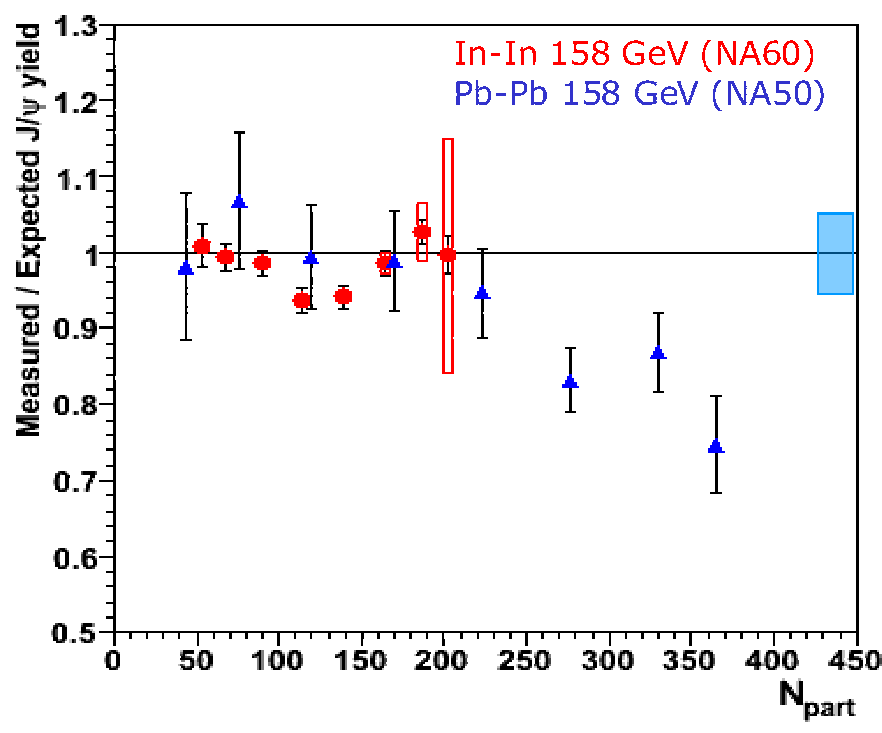

Figure 1: The comparison of NA50 and NA60 results versus $N_{\text {part }}$.

\section{The $\mathbf{J} / \psi$ production at the collider $\mathrm{RHIC}$}

The measurements of $\mathrm{J} / \psi$ production in $\mathrm{p}-\mathrm{p}, \mathrm{d}-\mathrm{Au}, \mathrm{Au}-\mathrm{Au}$ and $\mathrm{Cu}-\mathrm{Cu}$ collisions at $\sqrt{s}=$ $200 \mathrm{GeV}$ energy were made by PHENIX experiment at RHIC collider $[6,7,8,9]$. The suppression of the $\mathrm{J} / \psi$ production was presented as a ratio of $\mathrm{J} / \psi$ production in A-A collisions to the $\mathrm{J} / \psi$ production in p-p collisions, normalized to the number of binary collisions, $N_{\text {coll }}$. This $R_{A A}$ ratio (the nuclear modification factor), where $R_{A A}=d N_{A A} / d y /\left(d N_{p p} / d y *<N_{\text {coll }}>\right.$ ), was used for the comparison of the experimental data versus centrality of collisions, multiplicity, number of participants, $N_{\text {part }}$, energy density, transverse momentum and so on. The suppression of the J/ $\psi$ production in Au-Au collisions in forward rapidity range $1.2<|y|<2.2$ was found to be stronger than suppression in rapidity range $|y|<0.35$. For the most central events the suppression of the $\mathrm{J} / \psi$ production reaches a value near $80 \%$ (fig.2).

There are several theoretical models which could describe experimental data with and without taking into account the regeneration of $\mathrm{J} / \psi$. However, the predictions for the $\mathrm{J} / \psi$ production at $\mathrm{LHC}$ 


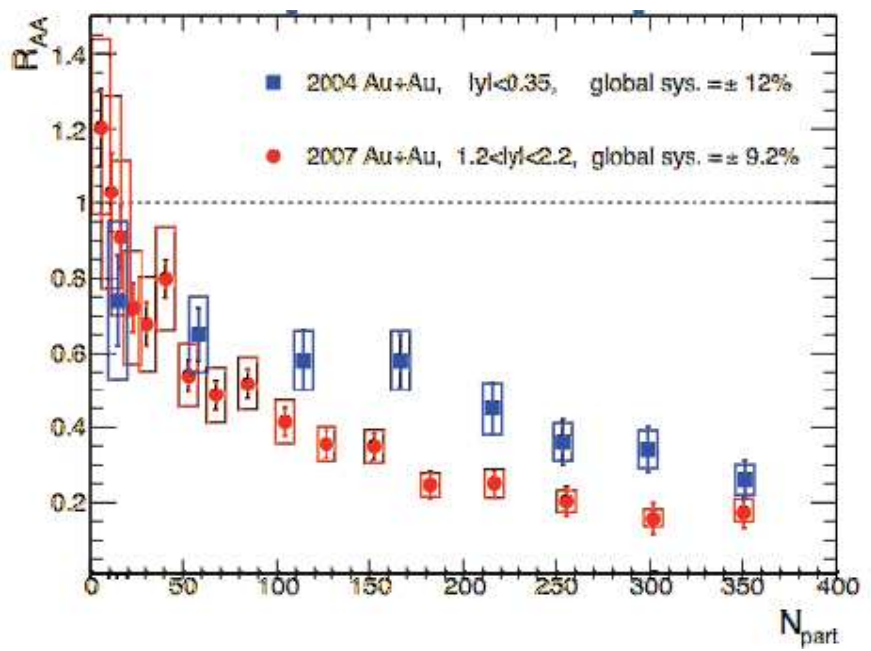

Figure 2: The nuclear modification factor $R_{A A}$ at mid- and forward rapidities versus $N_{\text {part }}$.

energy are different - from large suppression in the model with sequential suppression [10] to the enhancement in model with $c \bar{c}$ recombination [11]. The $\mathrm{J} / \psi$ production in Au-Au collisions at large transverse momentum $p_{\mathrm{T}}>5 \mathrm{GeV} / \mathrm{c}$ for mid-rapidity range was also measured by STAR experiment at RHIC. The suppression for the $\mathrm{J} / \psi$ production was smaller than measured at PHENIX and was near $50 \%$ for the most central collisions [12]. For extractions of the CNM effect, the PHENIX data for $\mathrm{Au}-\mathrm{Au}$ and $\mathrm{d}-\mathrm{Au}$ collisions were analyzed simultaneously. It was suggested that in $\mathrm{p}-\mathrm{p}, \mathrm{p}-\mathrm{A}$ and d-Au collisions the hot and dense nuclear matter was not formed and the normal suppression of charmonium production was produced only by cold nuclear matter. The nuclear modification factor for cold nuclear matter $R_{A A}(C N M)$ in Au-Au collisions was obtained. The ratio $R_{A A} / R_{A A}(C N M)$ shows the "anomalous" suppression of the $\mathrm{J} / \psi$ production in the hot and dense nuclear matter produced in relativistic heavy ions collisions. At $\sqrt{s}=200 \mathrm{GeV}$ the $R_{A A} / R_{A A}(C N M)$ ratio is approximately equal for different ranges of rapidity [13]. From the dependence of NA50, NA60 and PHENIX data on multiplicity it is seen that at RHIC energy "anomalous" suppression of $\mathrm{J} / \psi$ for all colliding nuclei is the same as at SPS energy. However, the suppression measured by PHENIX is stronger than suppression for $\mathrm{Pb}-\mathrm{Pb}$ collisions at SPS by using the dependence of "anomalous" $\mathrm{J} / \psi$ suppression on $N_{\text {part }}$ (fig3). So it is important to understand the reason of this effect and to choose the correct parameter for comparison the data.

\section{Quarkonium production at the LHC}

The LHC collider at CERN now gives the possibility to measure quarkonium production at the new energy range more than ten times higher than at RHIC. In experiments ALICE [14], ATLAS [15], CMS [16] and LHCb [17] at LHC, the charmonium production was measured in different rapidity and transverse momentum ranges. The $\mathrm{J} / \psi$ production during years $2010-2012$ was measured in p-p collisions at $2.76 \mathrm{TeV}, 7 \mathrm{TeV}$ and $8 \mathrm{TeV}$ and in $\mathrm{Pb}-\mathrm{Pb}$ collisions at $2.76 \mathrm{TeV}$. At the beginning of 2013 year it is planned to measure $\mathrm{p}-\mathrm{Pb}$ collisions for detailed investigation of 

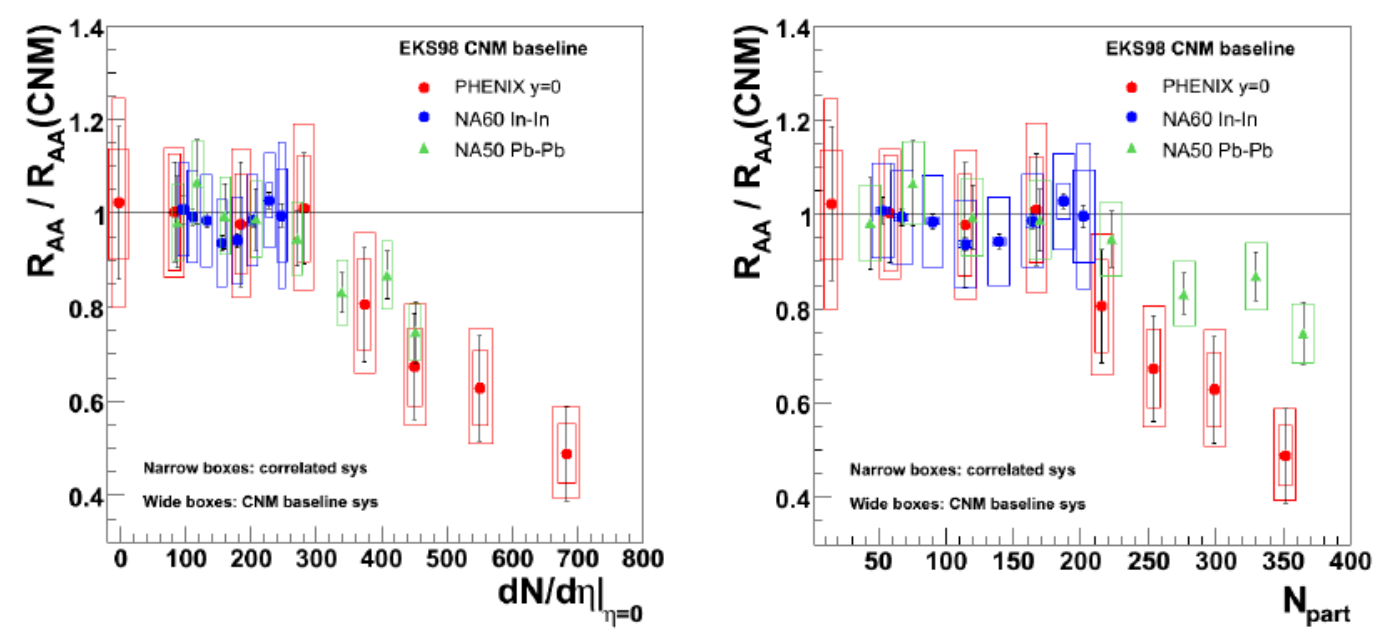

Figure 3: The comparison of NA50, NA60 and PHENIX $R_{A A}$ results versus multiplicity (left panel) and versus $N_{\text {part }}$ (right panel).

the cold nuclear matter effects. The first test measurements of $\mathrm{p}-\mathrm{Pb}$ collisions in 2012 were carried out successfully.

\subsection{Charmonium production in p-p collisions}

Measurement of the $\mathrm{J} / \psi$ production in $\mathrm{p}-\mathrm{p}$ collision at the same energy as in $\mathrm{Pb}-\mathrm{Pb}$ collision, provides the baseline for extracting the nuclear modification factor $R_{A A}$. The mechanism of $\mathrm{J} / \psi$ production is investigated and the contribution of B- decay to $\mathrm{J} / \psi$ production is measured. This contribution depends on rapidity and increases for larger values of $\mathrm{J} / \psi$ transverse momentum. The contribution of $\mathrm{B}$ - decay to $\mathrm{J} / \psi$ production cross section is approximately $10 \%$ for $p_{\mathrm{T}}$ near $1.5 \mathrm{GeV} / \mathrm{c}$ [17]. There is a good agreement for p-p collision between the data obtained by ALICE, LHCb, CMS and ATLAS experiments in the same kinematical domains [18]. The $\mathrm{J} / \psi$ production cross section depends on rapidity. For forward rapidity the cross section is smaller. For high energy the mean transverse momentum and production cross section of $\mathrm{J} / \psi$ are increased.

\subsection{Charmonium production in $\mathrm{Pb}-\mathrm{Pb}$ collisions}

Charmonium production in $\mathrm{Pb}-\mathrm{Pb}$ collisions at $2.76 \mathrm{TeV}$ was measured at LHC in ALICE, CMS and ATLAS experiments. In ALICE experiment the transverse momentum of inclusive $\mathrm{J} / \psi$ mesons was measured from values near zero up to $8 \mathrm{GeV} / \mathrm{c}$ in rapidity range $|y|<0.9$ (for $\mathrm{J} / \psi$ decay into two electrons) and $2.5<y<4$ (for muon channel). In ATLAS and CMS experiments charmonium production was measured in the rapidity range $|y|<2.4$, but the range of transverse momentum values depended on rapidity. In ATLAS experiment only $\mathrm{J} / \psi$ mesons with large transverse momentum $p_{\mathrm{T}}>6.5 \mathrm{GeV} / \mathrm{c}$ were measured $[15,16]$.

The $R_{A A}$ dependence on centrality in ALICE experiment is not so strong, as in PHENIX. In ALICE for the most central events and $2.5<y<4$ range the $R_{A A}$ value is more than two times larger than measured by PHENIX, so the suppression of $J / \psi$ production is smaller [19]. Also a 
smaller nuclear suppression is seen at ALICE at mid-rapidity in comparison with RHIC data [20]. The comparison of $R_{A A}$ from PHENIX and ALICE data is shown in the fig.4.
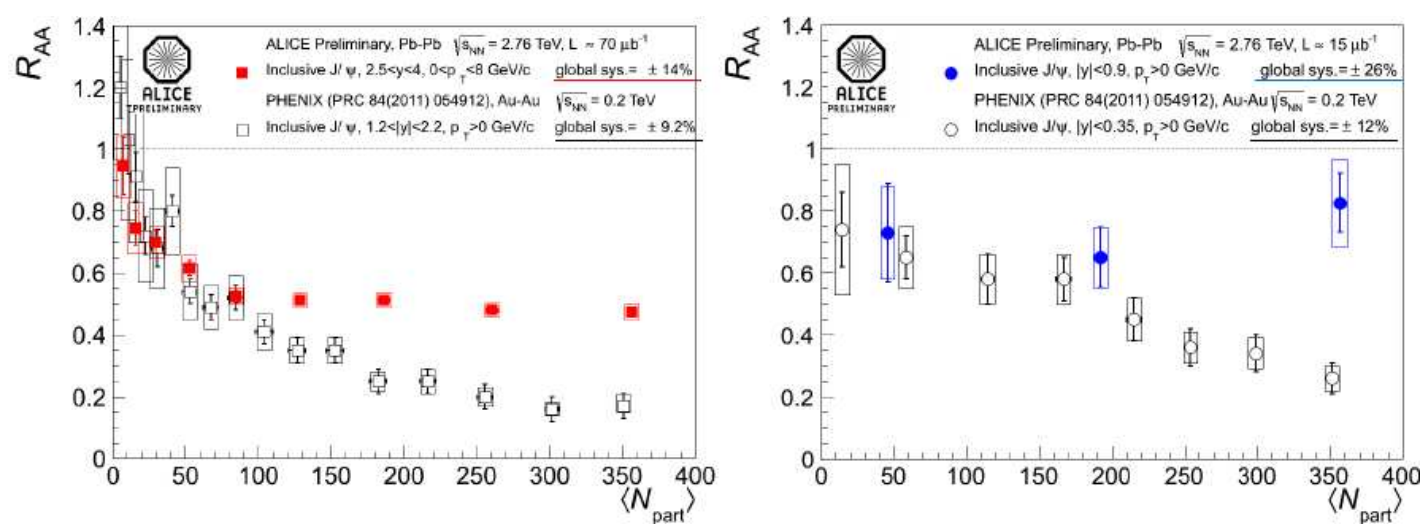

Figure 4: The comparison of PHENIX and ALICE $R_{A A}$ results. The two panels show the data at forward rapidity (left) and mid-rapidity (right).

But in CMS experiment [21] the suppression of "prompt" $\mathrm{J} / \psi$ production with high $p_{\mathrm{T}}$ (directly produced and obtained from feed-down decay) for the most central event is approximately equal to the suppression measured at RHIC. This points to an enhancement production of $\mathrm{J} / \psi$ at low $p_{\mathrm{T}}$ compared to high $p_{\mathrm{T}}$. It is also seen in ALICE data in different transverse momentum ranges (fig5). The suppression is stronger for higher transverse momentum.

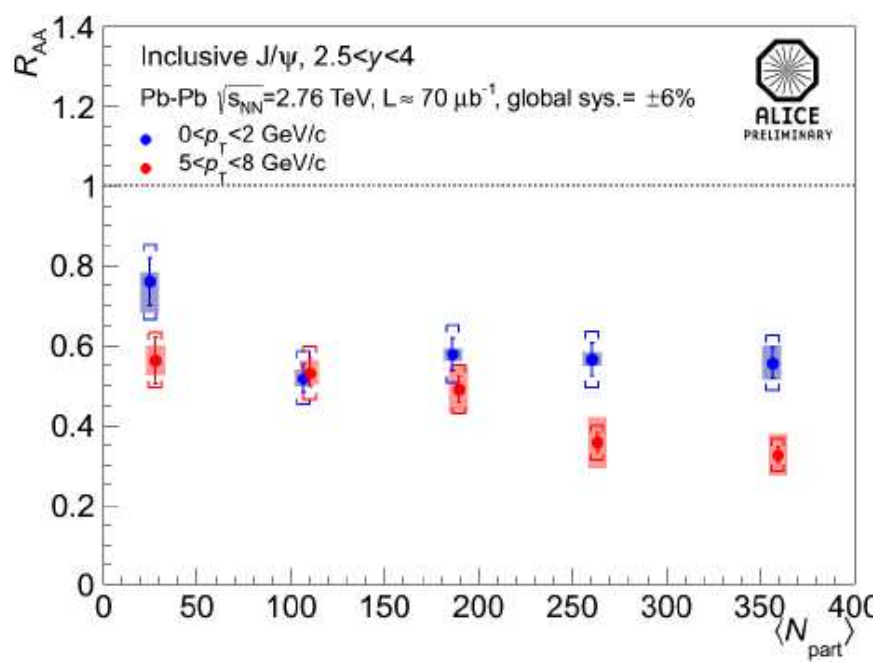

Figure 5: The ALICE $R_{A A}$ results in two different transverse momentum ranges versus $N_{\text {part }}$.

The $R_{A A}$ centrality dependence, measured in ALICE, was compared with a statistical hadronization model [22] and with transport models calculations [23, 24]. These models show a good agreement with the RHIC data. Both models predict the larger values for $R_{A A}$ at LHC than at RHIC in agreement with ALICE experimental data. The importance of $\mathrm{J} / \psi$ regeneration process is seen from comparison of ALICE experimental data with transport models (fig6). 

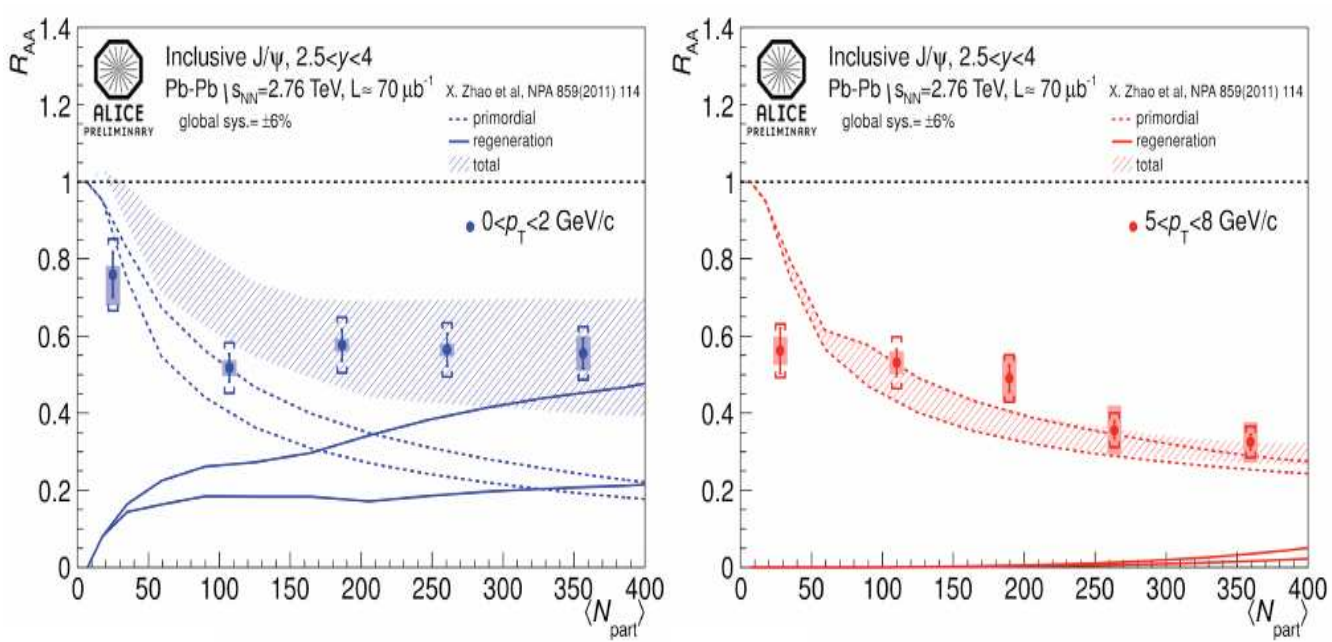

Figure 6: The comparison of ALICE $R_{A A}$ results with theoretical transport models, for forward rapidity (left panel) and mid-rapidity (right panel).

At low $p_{\mathrm{T}}$ values the contribution of $\mathrm{J} / \psi$ regeneration process consists near $50 \%$, while this contribution is negligible at large $p_{\mathrm{T}}$.

The $\mathrm{J} / \psi$ mean $p_{\mathrm{T}}$ dependence on centrality, measured by ALICE, has different behaviour than measured at lower energy. While mean $p_{\mathrm{T}}$ increases with $N_{\text {part }}$ at SPS and RHIC, it decreases at LHC. It confirms the obsevation that $\mathrm{J} / \psi$ with low $p_{\mathrm{T}}$ are less suppressed in central collisions at LHC. The comparison of $R_{A A} p_{\mathrm{T}}$ dependence, measured by ALICE,CMS and PHENIX is shown in fig.7. At low $p_{\mathrm{T}}$ region the suppression at LHC is less than at PHENIX and it grows with increasing the transverse momentum.

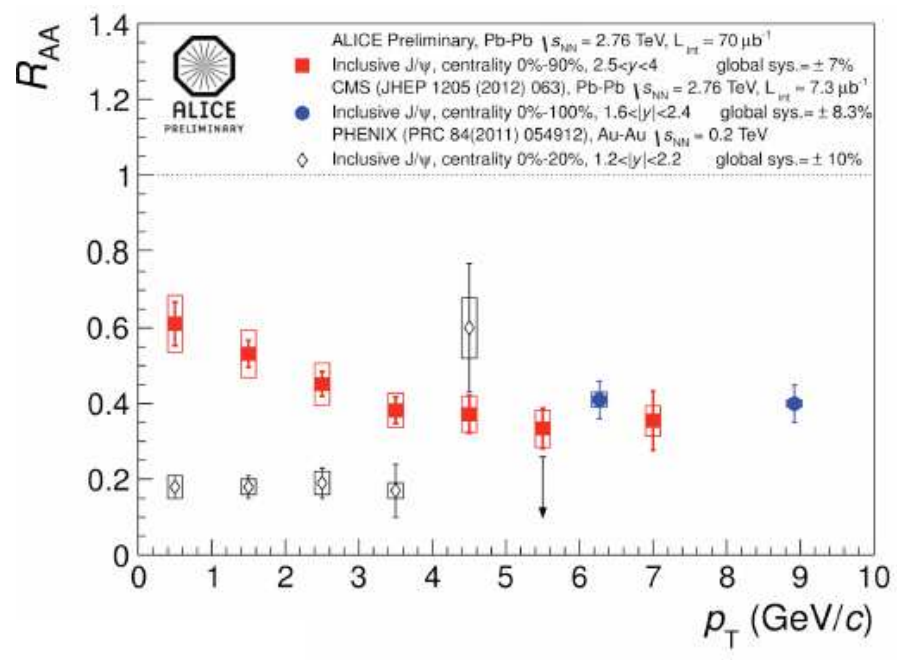

Figure 7: The comparison of $R_{A A} p_{\mathrm{T}}$ dependence, measured by ALICE, CMS and PHENIX.

The future measuring of cold nuclear matter effects in $\mathrm{p}-\mathrm{Pb}$ collisions is very important for the 
interpretation of the suppression results.

\section{Quarkonium production at fixed target at LHC beams}

Progress in our understanding of nuclear matter requires systematical and high statistical measurement also in low energy region. It is important to study the mechanism of quarkonium production and suppression at low energies up to $35 \mathrm{GeV}$ /per nucleon (SIS300) at the FAIR in CBM and at NICA collider in Dubna with high statistics. In order to understand the problem of quarkonium suppression it is necessary to measure $\mathrm{p}-\mathrm{p}, \mathrm{p}-\mathrm{A}$ and $\mathrm{Pb}-\mathrm{Pb}$ collisions in the same kinematical domains.

The energy intervals between AGS, SPS, RHIC and LHC are very important to study the mechanism of quarkonium production and suppression, in order to investigate medium effects and conditions of quark-gluon plasma formation. However, the luminosity in collider experiments at RHIC decreases with energy reduction and beam energy scan at RHIC produced data with low statistic and large systematic errors. If the proton and ion beams would be used at LHC with fixed targets, the energy interval between SPS and RHIC in p-A and A-A collisions could be covered. For $7 \mathrm{TeV}$ proton beam, the energy in N-N c.m. is $\sqrt{s}=114.6 \mathrm{GeV}$, for Pb beam at $2.75 \mathrm{TeV}$ it is $\sqrt{s}=71.8 \mathrm{GeV}$. Using LHC beams with reduced energy it would be possible to scan energy range between SPS and RHIC [25]. This is a unique possibility to clarify the mechanism of charmonium, $\mathrm{J} / \psi$ and $\psi^{\prime}$ production, to separate two possibilities: i): hard production and suppression in QGP and/or hadronic dissociation or ii): hard production and secondary statistical production with recombination, since the probability of recombination decreases with decreasing energy of collision in thermal model. In order to study the feasibility of using the fixed target at LHC for charmonium production, the geometrical acceptances for $\mathrm{J} / \psi$ production on fixed target by means of AliRoot - FAST simulations were obtained. As described in [25] for quarkonium production the phenomenological Colour Evaporation Model (CEM) was used. The rapidity and transverse momentum distributions for "prompt" $\mathrm{J} / \psi$ production were obtained respectively as a parameterization of the CEM predictions and by extrapolating to LHC energy the $J / \psi$ transverse momentum $p_{\mathrm{T}}$ distribution, measured at mid-rapidity by the CDF experiment at c.m. energy near $1.8 \mathrm{TeV}$. The $30000 \mathrm{~J} / \psi$ events were generated and geometrical acceptances of the ALICE dimuon spectrometer were obtained for $\mathrm{J} / \psi$, produced in $\mathrm{Pb}-\mathrm{Pb}$ collisions at $\sqrt{s}=5.5 \mathrm{TeV}$ in N-N c.m. system and in p-p collisions at $\sqrt{s}=14 \mathrm{TeV}$ in agreement with the existing calculations [26]. In the same frame the geometrical acceptances of $\mathrm{J} / \psi$ production for PHENIX at RHIC and fixed target experiments NA50 at SPS and HERA-B were calculated for comparison.

The calculated geometrical acceptances for fixed target measurement are of the same order and even larger than geometrical acceptances for colliding nuclei in ALICE. The counting rates are calculated and it is shown that the $\mathrm{J} / \psi$ production on fixed targets at LHC could be measured with high statistics collected in several days of data taking [25].

\subsection{Luminosity, cross sections and counting rates for $\mathrm{p}-\mathrm{p}, \mathrm{p}-\mathrm{A}$ and $\mathrm{Pb}-\mathrm{Pb}$ collisions}

As it was already used for the experiment on collider with a fixed target at HERA-B [27], the target in the form of thin ribbon could be placed around the main orbit of LHC. The life time of the beam is determined by the beam-beam and beam-gas interactions. Therefore after some 
time the particles will leave the main orbit and interact with the target ribbon. So for fixed target measurements only halo of the beam will be used. Therefore no deterioration of the main beam will be introduced. The experiments at different interaction points (IP) will not feel any presence of the fixed target at the IP of ALICE. The luminosity estimate is shown in the Table 2. It is calculated for $1.15 \cdot 10^{11}$ protons per bunch, 44 bunches and life time 15.4 hours. From these parameters we get particle loss of $3.2 \cdot 10^{12}$ during one hour and luminosity about $1.5 \cdot 10^{29}$ for 500 micron lead ribbon.

Since the target ribbon should not interfere during the beam formation and acceleration process it should be lifted in the working position after the tuning of the beam. The value of the nucleonnucleon charmonium total production cross section shown in the Table 2 for $14 \mathrm{TeV}$ was calculated by CEM model with MRST HO PDF. The cross sections for lower energies were obtained by interpolation of the measured at RHIC proton-proton collision at $\sqrt{s}=200 \mathrm{GeV}$, from NA51 p-p and p-d experiment at $450 \mathrm{GeV}$ per nucleon $(\sqrt{s}=29.1 \mathrm{GeV})$ and extracted from the data of NA50 experiment at $\sqrt{s}=27.4 \mathrm{GeV}$ for proton-lead collisions.

Table 1: Luminosity, cross sections $\left(x_{F}>0\right)$ and counting rates.

\begin{tabular}{|c|c|c|c|c|c|c|c|}
\hline System & $\sqrt{s}, \mathrm{TeV}$ & $\sigma_{n n}, \mu \mathrm{b}$ & $\begin{array}{c}\sigma_{p A}, \mu \mathrm{b} \\
\left(A^{0.92} \sigma_{n n}\right)\end{array}$ & $\mathrm{I}, \%$ & $\mathrm{IB} \sigma_{p A}, \mu \mathrm{b}$ & $\mathrm{L}, \mathrm{cm}^{-2} s^{-1}$ & Rate, $h^{-1}$ \\
\hline $\mathrm{pp}$ & 14 & 32.9 & 32.9 & 4.7 & 0.091 & $5 \cdot 10^{30}$ & 1635 \\
$\mathrm{pp}_{\text {RHIC }}$ & 0.200 & 2.7 & 2.7 & 3.59 & 0.0057 & $2 \cdot 10^{31}$ & 410 \\
$\mathrm{pP}_{\text {NA50 }}$ & 0.0274 & 0.19 & 25.7 & 14.0 & 0.212 & $7 \cdot 10^{29}$ & 535 \\
$\mathrm{pPb}_{\text {fixed }}$ & 0.1146 & 0.65 & 80.2 & 5.98 & 0.310 & $1 \cdot 10^{29}\left(^{\star}\right)$ & 112 \\
$\mathrm{pPb}_{\text {fixed }}$ & 0.0718 & 0.55 & 74.6 & 7.97 & 0.349 & $1 \cdot 10^{29}$ & 126 \\
$\mathrm{PbPb}_{\text {fixed }}$ & 0.0718 & 0.55 & 11970 & 7.97 & 42.9 & $2.2 \cdot 10^{27}\left(^{\star \star}\right)$ & 378 \\
\hline
\end{tabular}

$\left.{ }^{\star}\right)-\mathrm{pPb}_{\text {fixed }}, 500 \mu$ wire, $3.2 \cdot 10^{12}$ protons $/ 60 \mathrm{~min}$.

$\left({ }^{\star}\right)-\mathrm{PbPb}_{\text {fixed }}, 500 \mu$ wire, $6.8 \cdot 10^{9}$ ions $/ 60 \mathrm{~min}$.

With the counting rate values presented in Table 2 for $\mathrm{p}-\mathrm{A}$ and A-A collisions with fixed target high statistical results could be obtained for possible measurements of $\mathrm{J} / \psi$ production. For A-A collisions luminosity is smaller but the production cross section is larger. Therefore the counting rate is of the same order. It is also clear that the measurement of $\psi^{\prime}$ production is feasible with better statistical accuracy than at RHIC collider.

Recenly the group of the French physicists has also suggested the fixed target experiment at LHC using beams extracted with strong crystalline field [28]. The fixed target experiment provides precise quarkonium studies and has advantage of high luminosity and possibility to make measurements on many nuclear targets, what is strongly limited at collider. The use of fixed target at LHC could provide in a short time the data for different targets and maybe for different projectile nuclei with high statistics. Therefore the important information about mechanism of charmonium production and possible QGP formation could be obtained.

The work was supported by the Russian Academy of Sciences and by the Russian Foundation for Basic Research ( grant N 12-02-91508-CERN-a ). 


\section{References}

[1] T. Matsui and H. Satz, Phys. Lett. B178, 416 (1986).

[2] B. Alessandro et al., NA50 collaboration, Eur. Phys. J C39, 335 (2005) and references therein.

[3] B. Alessandro et al., NA50 collaboration, Phys. Lett. B553, 167 (2003).

[4] R. Arnaldi et al., NA60 collaboration, Nucl. Phys. A830, 345c (2009); Eur. Phys. J C59, 607 (2009).

[5] K.J. Eskola et al., Eur. Phys. J C9, 61 (1999).

[6] A. Adare et al., PHENIX collaboration, Phys. Rev. Lett. 98232301 (2007).

[7] A. Adare et al., PHENIX collaboration, Phys. Rev. C77 024912 (2008).

[8] A. Adare et al., PHENIX collaboration, arXiv :1103.6269 v1.

[9] A. Adare et al., PHENIX collaboration, arXiv :1105.1960.

[10] R. L. Thews et al., Eur. Phys. J C43, 97 (2005); R. L. Thews and M.I. Mangano, Phys. Rev. C73, 014904 (2006).

[11] A. Andronic, P. Braun-Munzinger, K. Redlich, and J. Stachel, Phys. Rev. Lett. 659, 149 (2008).

[12] H. Masui and Z. Tang, STAR collaboration, J. Phys. G38, 124002 (2011).

[13] H. Brambilla et al., Eur. Phys. J C71, 1534 (2011).

[14] K. Aamodt et al., ALICE collaboration, Phys. Lett. B704, 442 (2011).

[15] G. Aad et al., ATLAS collaboration, Nucl. Phys. B 850, 387 (2011); arXiv:1012.5419 v1.

[16] S. Chatrchyan et al., CMS collaboration, JHEP 05, 063 (2012).

[17] R. Aaij et al., LHCb collaboration, Eur. Phys. J C71, 1645 (2011); arXiv:1103.0423 v2.

[18] P. Pillot, ALICE collaboration, J. Phys. G38, 124111 (2011).

[19] B. Abelev et al., ALICE collaboration, Phys. Rev. Lett. 109, 072301 (2012).

[20] G.M. Garcia, ALICE collaboration, J. Phys. G38, 124034 (2011).

[21] T. Dahms, CMS collaboration, J. Phys. G38, 124105 (2011).

[22] A. Andronic et al., J. Phys. G38, 124081 (2011).

[23] X. Zhao and R. Rapp, Nucl. Phys. A859, 1214 (2011).

[24] Y. Liu et al., Phys. Lett. B678, 72 (2009).

[25] A. B. Kurepin, N. S. Topilskaya and M. B. Golubeva, Phys. Atom. Nucl. 74,446 (2011) and references therein.

[26] ALICE Physics Performance Report, vII, 558 (2005).

[27] K. Ehret, Nucl. Instr. Meth. A446, 190 (2000).

[28] J.P. Lansberg et al., arXiV 1202.6585. 